\title{
Synthesis and Biological Evaluation of Isatin Based Thiazole Derivatives
}

\author{
Tariq Aziz*1, Fazal Rahim², Roh Ullah ${ }^{3}$, Asmat Ullah4 ${ }^{4}$, Fazal Haq ${ }^{5}$, Farman Ullah Khan ${ }^{6}$, Mehwish \\ Kiran $^{7}$, Noor Saeed Khattak ${ }^{8}$ and Mudassir Iqbal ${ }^{9}$ \\ ${ }^{1}$ College of Chemical and Biological Engineering, Zhejiang University, China \\ ${ }^{2}$ Department of Chemistry, Hazara University, Pakistan \\ ${ }^{3}$ School of Chemistry and Chemical Engineering, Beijing Institute of Technology, China \\ ${ }^{4}$ School of Pharmacy Xi'an Jiaotong university Shaanxi, China \\ ${ }^{5}$ College of Chemical and Biological Engineering, Zhejiang University, China \\ ${ }^{6}$ Department of Chemistry, University of Science and Technology Bannu 28000, Pakistan/ Department of Chemistry, University \\ of Lakki Marwat, Pakistan
}

${ }^{7}$ Department of Horticulture Gomal University, Dera Ismail Khan, KPK, Pakistan

${ }^{8}$ Islamia College University Peshawar, Pakistan

${ }^{9}$ College of Chemical and Biological Engineering, Zhejiang University, Hangzhou, China

*Corresponding author: Tariq Aziz, College of Chemical and Biological Engineering, Zhejiang University, Hangzhou 310027, China

ARTICLE INFO

Received: 慧 June 29, 2020

Published: 幽 July 10, 2020

Citation: Tariq Aziz, Fazal Rahim, Roh Ullah, Asmat Ullah, Fazal Haq, et al., Synthesis and Biological Evaluation of Isatin Based Thiazole Derivatives. Biomed J Sci \& Tech Res 28(5)-2020. BJSTR. MS.ID.004704.
ABSTRACT

Isatin is an important class of nitrogen containing heterocyclic aromatic compounds, found in plants, human blood and tissue, and considered as very attractive compound in medicinal chemistry. On the other hand, thiazole heterocyclic derivatives are other important key compounds received wide attention in pharmaceutical industry. In this study, we have synthesized series of isatin based thiazloe derivatives, elucidated their structures with different spectroscopic techniques, such as FTIR and Proton nuclear magnetic resonance spectroscopy and screened for Lipoxygenase inhibitory potential.

Keywords: Isatin; Thiazole; Lipoxygenase; Heterocyclic; Biological importance

\section{Introduction}

Isatin is an important class of nitrogen containing heterocyclic aromatic compounds, found in plants, human blood and tissue and acts as important specie for the synthesis of various heterocyclic compounds especially, indolic and quinolinic compounds [1]. Isatinbased multicomponent reactions are one of the preferred strategies for the one pot synthesis of spirooxindole fused heterocycles [2]. Isatin (1H-indole-2,3-dione) derivatives show a broad spectrum of biological activities such as antibacterial, antifungal, antiviral, anti-inflammatory, anti-convulsant and anticancer drug [3,4]. Some of the Istin derivatives have been approved for clinical uses. Its derivatives represent an important class of heterocyclic compounds that can be used as precursors for drug synthesis [5]. Due to the importance of this derivative and its wide range of applications, it is justified to apply the "Lord of the Rings" of aromatic compounds. Therefore, designing attractive new decorative structures remains a positive challenge and a goal of many research groups $[6,7]$. Isatin moiety is responsible for a wide spectrum of biological property such as anticancer and antiviral in many synthetically versatile molecules [8]. Isatin derived ketimines represent readily available synthetic precursors of the 3-amino-2-oxindole moiety via enantioselective Mannich type reactions $[9,10]$. Another indolebased moiety with proven anti-proliferative potential via inhibition of tyrosine kinases like VEGFR-1, VEGFR-2 and cyclic dependent kinases, an isatin analogue has been approved by the FDA for the 
treatment of gastrointestinal stromal tumors and advanced renal cell carcinoma [11].

Bis-Schiff bases of isatin were found to possess significant anti-viral, antibacterial and anti-fungal activity [12]. The azetidine and thiadiazine derivatives of isatin were also found to possess significant antibacterial activity [13]. 5-Nitro-1H-indole-2,3dione3-N-(4H-methyl phenyl) thiosemicarbazones screened for antibacterial activity against E.coli and S. aureus by the cup-plate method and found active [14]. Meshram et al. also developed an isatin-based multicomponent reaction for the synthesis of spirooxindole fused N-heterocyles with anticancer properties [2]. Zhang et al. first reported the vinylogous, henry reaction between isatin and 3,5-dialkyl-4-nitroisoxazole, leading to isoxazolesubstituted 3-hydroxy-2-oxindole derivatives, medicinally important compounds [15], also provided the basis for removal of industrial dye wastewater $[16,17]$. Isatin based hydrazones are a class of compounds that have been largely used as synthons for the synthesis of heterocyclic compounds because of the presence of various reactive centers in the moiety [18]. Isatin derived propargylic alcohols have been extensively used as versatile synthons in organic synthesis as they incorporate both triple bonds and hydroxyl functional groups [19]. A variety of catalytic enantioselective addition reactions to isatin-derived ketimines have been developed, including Strecker reactions, aza-Henry reactions and aza-Friedel-Crafts reactions etc. [20]. Spirooxindoles, the 3-substituted oxindole unit is recognized as an important structural motif that forms the core of numerous heterocyclic compounds and has a wide spectrum of pharmaceutical activity, such as being a gastrin/CCK-B receptor antagonist, a vasopressin receptor antagonist and antimalarial agent [21]. In this study, here we have discussed in detail the synthesis of isatin and their derivatives particularly in medicinal fields, because isatin plays a key effect like ascorbic acid, hydroxyl amino acids, omeprazole, thiol compounds and imidazole etc.; which is helpful for the pathogenesis of gastric ulcer and peptic ulcer. Also discuss antioxidants, which are responsible for converting free radicals into stable substances, especially serious diseases caused by diabetes. We can say, that isatin provides a critical and dynamic role in the pharmaceutical industry.

\section{Synthetic Scheme}

Figure 1.

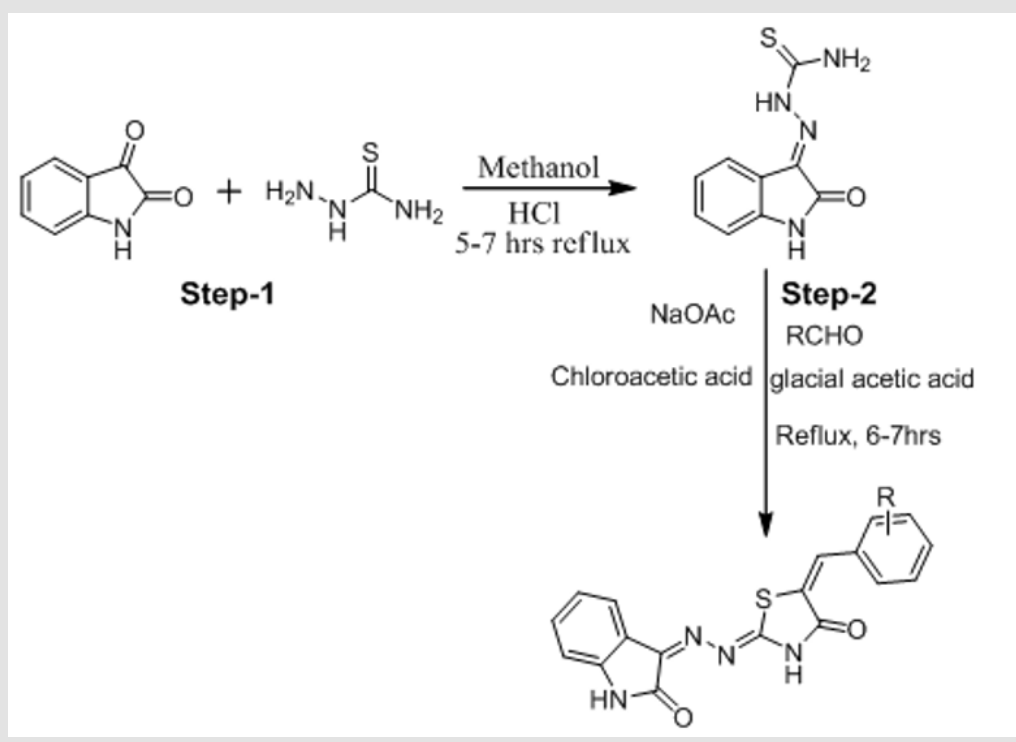

Figure 1: Synthesis of isatin based thiazole derivatives.

\section{Experimental}

\section{Materials and Methods}

The synthesis of isatin derivatives was carried out in two-step reactions. In first step, thiosemicarbazide (1 mmol) was mixed with isatin in methanol as a solvent in the presence of $\mathrm{HCl}$ as a catalyst and refluxed for 5-7 hrs. Reaction completion was checked by thin layer chromatography. After reaction completion the crude mixture was filter and then washes with methanol. The solid ppt of pure products was obtained. In second step, the product obtained in the first step ( $1 \mathrm{mmol}$ ) was mixed with chloro acetic acid, sodium acetate and aromatic aldehydes ( $1 \mathrm{mmol}$ ) in the presence of glacial acetic acid and refluxed for 6-7 hrs. Reaction completion was checked by thin layer chromatography. After reaction completion, the mixture was filter then wash with glacial acetic acid to obtained pure solid products. The structures of all derivatives (77-89) were confirmed by different spectroscopic techniques, such as $1 \mathrm{H}$ NMR and mass Spectroscopy. In the present work, all the solvents were used of analytical grade without further distillation. i.e. isatin $\left[\mathrm{C}_{8} \mathrm{H}_{5} \mathrm{NO}_{2}\right]$ (s), thiosemicarbazide $\left[\mathrm{H}_{2} \mathrm{NNHC}(=\mathrm{S}) \mathrm{NH}_{2}\right](\mathrm{s})$, methanol[MeOH] (l), sodium acetate $\left[\mathrm{C}_{2} \mathrm{H}_{3} \mathrm{NaO}_{2}\right](\mathrm{s})$, chloro acetic acid $\left[\mathrm{C}_{2} \mathrm{H}_{3} \mathrm{O}_{2} \mathrm{Cl}\right]$ $(\mathrm{s})$, ethanol (EtOH), Potassium Carbonate $\left[\mathrm{K}_{2} \mathrm{CO}_{3}\right](\mathrm{s})$, Distilled 
water, acetone, and n-hexane were used as such without further distillation. All these chemicals were found 98-99.9\% pure. The substrate are used different aromatic aldehydes such as (2-Nitro benzaldehyde), (4-Benzyaloxy-3 methoxy benzaldehyde), (4-Nitro benzaldehyde), (Terepthaldehyde), (4-Hydroxy benzaldehyde), (3,5-Dichloro salicyldehyde), (Syringeldehyde), (3-Nitro benzaldehyde), (3-Hydroxy benzaldehyde), (2,3-dimethoxy benzaldehyde), (3-chloro,4-hydroxy benzaldehyde). The reagents used were glacial acetic acid (Merck).

\section{Characterizations}

$1 \mathrm{H}$ nuclear magnetic resonance (1H NMR) spectra were recorded on a Bruker Advanx-400 DMX NMR spectrometer using D20 as a solvent. Chemical shift values are given in $\delta$ (ppm).
Electron ionization (EI), and high resolution electron ionization mass spectra (HREIMS) were recorded on advance jeol JMS-600H apparatus. Pre-coated silica gel aluminum plates (kieselgel 60, 254, E. merck, Germany) were used for monitoring of reactions. By UV lamp of 254 and $365 \mathrm{~nm}$, the spot were visualized.

\section{Results and Discussions}

Table 1 (2Z,5E)-5-(2-nitrobenzylidene)-2-[22]-(2-oxoinodolin3ylidene) hydrazono)thiazolidin-4-one (1)

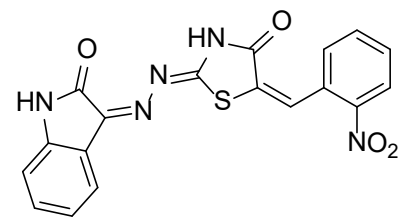

Table 1: Structure of all synthetic Isatin derivatives (77-89).

\begin{tabular}{|c|c|c|c|}
\hline S. No. & $\mathbf{R}$ & S. No. & $\mathbf{R}$ \\
\hline 77 & & 84 & \\
\hline 78 & & 85 & \\
\hline 79 & & 86 & \\
\hline 80 & & 87 & \\
\hline 81 & & 88 & \\
\hline 82 & & 89 & \\
\hline 83 & & & \\
\hline
\end{tabular}

Formula, $\mathrm{C}_{18} \mathrm{H}_{11} \mathrm{~N}_{5} \mathrm{O}_{4} \mathrm{~S}$;

Yield: 74\%; ${ }^{1} \mathrm{H}-\mathrm{NMR}$ : (DMSO- $d_{6}, 300 \mathrm{MHz}$ ): $\delta 12.73$ (s, 1H, NH), 7.8 (m, 2H, H-2', CH aldehydic), 7.6 (d, 2H, $J_{4,5 / 7,6}=7.2 \mathrm{~Hz}, \mathrm{H}-4 / 7$ ), 7.3(m, 2H, H-5,6). 7.1(m, 3H, H-4',5',6'), EI-MS: $m / z$ (rel. int. \%): 393(M+100), 220 (84) 192 (56), 132 (27).

$(2 Z, 5 E)-5-(4-(3-m e t h o x y b e n z y l o x y)$ benzylidene $)-2-\{(Z)-(2-$ oxoindolin-3 ylidene)hydrazono)thiazolidin-4-one (2)<smiles>COc1cccc(COc2ccc(/C=C3\S/C(=N\N=C4\C(=O)Nc5ccccc54)NC3=O)cc2)c1</smiles> 
Formula, $\mathrm{C}_{26} \mathrm{H}_{20} \mathrm{~N}_{4} \mathrm{O}_{4} \mathrm{~S}$;

Yield: 74\%; ${ }^{1} \mathrm{H}-\mathrm{NMR}$ : (DMSO- $d, 300 \mathrm{MHz}$ ): $\delta 12.7$ (s, NH, $1 \mathrm{H}), 10.6(\mathrm{~s}, \mathrm{NH}, 1 \mathrm{H}), 8.1\left(\mathrm{~s}, 1 \mathrm{H}, \mathrm{CH}\right.$ aldehydic), $7.8\left(\mathrm{~d}, 2 \mathrm{H}, J_{4,5 / 7,6}\right.$ $=7.2 \mathrm{~Hz}, \mathrm{H}-4 / 7$ ), 7.6 (d, 2H, $\left.J_{2^{\prime} 3^{\prime} 6^{\prime}, 5^{\prime}}=8.1 \mathrm{~Hz}, \mathrm{H}-2^{\prime \prime}, 6^{\prime \prime}\right), 7.4$ (m, 4H, H-3'/5'/5/6), 7.2 (s, 1H, H-2"), 7.1 (m, 3H, H-4",5",6"), 3.6 (s, 2H, $\mathrm{OCH}_{2}$ ). EI-MS: $m / z$ (rel. int. \%): 484(m+ 46),220 (87), 192(100), $150(24), 104(38)$.

$(2 Z, 5 E)-5-(4-n i t r o b e n z y l i d e n e)-2-\{(Z)-(2-o x o i n d o l i n-3-$ ylidene)hydrazono)thiazolidin-4-one (3)<smiles>O=C1N/C(=N/N=c2/[nH]c(=O)[nH]c3ccccc23)S/C1=C\c1ccc([N+](=O)[O-])cc1</smiles>

Formula, $\mathrm{C}_{18} \mathrm{H}_{11} \mathrm{~N}_{5} \mathrm{O}_{4} \mathrm{~S}$;

Yield: 84\%; ${ }^{1} \mathrm{H}-\mathrm{NMR}$ : (DMSO- $d_{6}, 300 \mathrm{MHz}$ ): $\delta 12.77(\mathrm{~s}, 1 \mathrm{H}, \mathrm{NH}$ ), $10.2(\mathrm{~s}, 1 \mathrm{H}, \mathrm{NH}), 8.1\left(\mathrm{~s}, 1 \mathrm{H}, \mathrm{CH}\right.$ aldehydic), $7.6\left(\mathrm{~d}, 2 \mathrm{H}, J_{3^{\prime} 2^{\prime} / 5^{\prime} 6^{\prime}}=7.5 \mathrm{~Hz}\right.$, H-3'/5'), 7.4 (d, 2H, $\left.J_{4,5 / 7,6}=7.4 \mathrm{~Hz}, \mathrm{H}-4 / 7\right), 7.3$ (m, 2H, H-5/6), 7.0(d, $\left.2 \mathrm{H}, \mathrm{H}-J_{2^{\prime} 3^{\prime} / 6,5^{\prime}}=7.5 \mathrm{~Hz}, \mathrm{H}-2^{\prime} / 6^{\prime}\right)$;

EI-MS: $m / z$ (rel. int. \%): 393(m+1 100), 192 (98), 220 (83), 176(20).

(2Z,5E)-5-(4-hydroxybenzylidene)-2-\{(Z)-(2-oxoindolin-3ylidene)hydrazono)thiazolidin-4-one (4)<smiles>O=C1N/C(=C\c2ccc(O)cc2)S/C1=N/N=C1/C(=O)Nc2ccccc21</smiles>

Formula, $\mathrm{C}_{18} \mathrm{H}_{12} \mathrm{~N}_{4} \mathrm{O}_{3} \mathrm{~S}$;

Yield: 82\%; ${ }^{1} \mathrm{H}-\mathrm{NMR}$ (DMSO- $d_{6}, 300 \mathrm{MHz}$ ): $\delta 12.7(\mathrm{~s}, 1 \mathrm{H}, \mathrm{NH})$, 10.2(s,1H, NH), 8.0 (s, $1 \mathrm{H}, \mathrm{CH}$ aldehydic), $7.6\left(\mathrm{~d}, 2 \mathrm{H}, J_{4,5 / 7,6}=7.4 \mathrm{~Hz}\right.$, $\mathrm{H}-4 / 7), 7.4(\mathrm{~m}, 2 \mathrm{H}, \mathrm{H}-5 / 6), 7.1\left(\mathrm{~d}, 2 \mathrm{H}, \mathrm{H}-J_{2^{\prime} 3^{\prime} / 6^{\prime} 5^{\prime}}=7.5 \mathrm{~Hz}, \mathrm{H}-2^{\prime} / 6^{\prime}\right)$, $7.0\left(\mathrm{~d}, 2 \mathrm{H}, J_{3^{\prime}, 2^{\prime} / 5^{\prime}, 6^{\prime}}=7.7 \mathrm{~Hz}, \mathrm{H}-3^{\prime} / 5^{\prime}\right)$.

EI-MS: $m / z$ (rel. int.\%):364(M+100), 192(89), $220(83), 176(27)$.

(2Z,5E)-5-(2,4-dichloro-5-hydroxybenzylidene)-2-\{(Z)-(2oxoindolin-3-ylidene)hydrazono)thiazolidin-4-one (5)

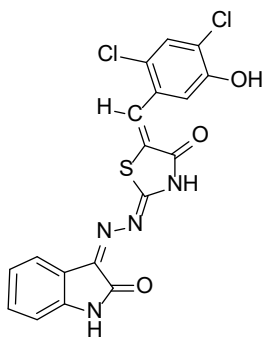

Formula, $\mathrm{C}_{18} \mathrm{H}_{10} \mathrm{Cl}_{2} \mathrm{~N}_{4} \mathrm{O}_{3} \mathrm{~S}$;

Yield: 82\%; ${ }^{1} \mathrm{H}-\mathrm{NMR}$ : (DMSO- $\left.d_{6}, 300 \mathrm{MHz}\right): \delta 12.7(\mathrm{~s}, 1 \mathrm{H}, \mathrm{NH})$, 10.1(s, 1H, NH), 8.0 (s, 1H, CH aldehydic), 7.6(s, 1H, H-3'), 7.4(d, 2H, $\left.J_{4,5 / 7,6}=7.4 \mathrm{~Hz}, \mathrm{H}-4 / 7\right), 7.1$ (m, 2H, H-5,6), 7.0(s, 1H, H-6');

EI-MS: $m / z \quad$ (rel. int. \%): 431( $\left.\mathrm{M}^{+}, \quad 100\right), \quad 192(98)$, 220(96),150(20),132(26).

$(2 Z, 5 E)-5$-(4-hydroxy-3,5-dimethoxybenzylidene)-2-((Z)-(2oxoindolin-3-ylidene)hydrazono)thiazolidin-4-one (6)

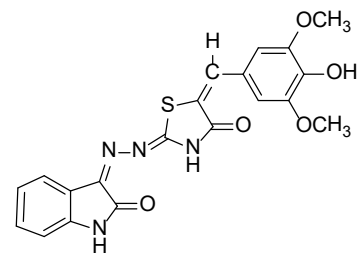

Formula, $\mathrm{C}_{20} \mathrm{H}_{16} \mathrm{~N}_{4} \mathrm{O}_{5} \mathrm{~S}$;

Yield: 82\%; ${ }^{1} \mathrm{H}-\mathrm{NMR}$ : (DMSO- $\left.d_{6}, 300 \mathrm{MHz}\right): \delta 12.6(\mathrm{~s}, 1 \mathrm{H}, \mathrm{NH})$, 10.4(s, $1 \mathrm{H}, \mathrm{NH}), 8.1$ (s, $1 \mathrm{H}, \mathrm{CH}$ aldehydic), $7.7\left(\mathrm{~d}, 2 \mathrm{H}, J_{4,5 / 7,6}=7.7 \mathrm{~Hz}\right.$, H-4/7), 7.5 (m, 2H, H-5,6), 7.3(s, 1H, H-2'), 7.2(s, 1H, H-6');

EI-MS: $m / z$ (rel. int. \%): 424(M+100), 220(92), 193(91), 151(29), 132(22).

$(2 Z, 5 E)-5$-(3-hydroxybenzylidene)-2-((Z)-(2-oxoindolin-3ylidene)hydrazono)thiazolidin-4-one (7)<smiles>O=C1NC(=NN=C2C(=O)Nc3ccccc32)SC1=Cc1cccc(O)c1</smiles>

Formula, $\mathrm{C}_{18} \mathrm{H}_{12} \mathrm{~N}_{4} \mathrm{O}_{3} \mathrm{~S}$;

Yield: 81\%; ${ }^{1} \mathrm{H}-\mathrm{NMR}$ : (DMSO- $\left.{ }_{6}, 300 \mathrm{MHz}\right): \delta 12.4(\mathrm{~s}, 1 \mathrm{H}, \mathrm{NH}$ ), 10.1(s,1H, NH), 8.1 (s, 1H, CH aldehydic), $7.4\left(\mathrm{~d}, 2 \mathrm{H}, J_{4,5 / 7,6}=7.5 \mathrm{~Hz}\right.$, $\mathrm{H}-4 / 7), 7.2(\mathrm{~m}, 2 \mathrm{H}, \mathrm{H}-5 / 6), 7.1\left(\mathrm{~d}, 1 \mathrm{H}, J_{2^{\prime} 3^{\prime}}=7.4 \mathrm{~Hz}, \mathrm{H}-2^{\prime}\right), 7.0(\mathrm{~m}, 3 \mathrm{H}$, $\left.\mathrm{H}-4^{\prime} / 5^{\prime} / 6^{\prime}\right)$.

EI-MS: $m / z$ (rel. int. \%): 364(M+1 , 78), 230(83), 182(82), 176(27).

(2Z,5E)-5-(2,3-dimethoxybenzylidene)-2-((Z)-(2-oxoindolin3-ylidene)hydrazono)thiazolidin-4-one (8)

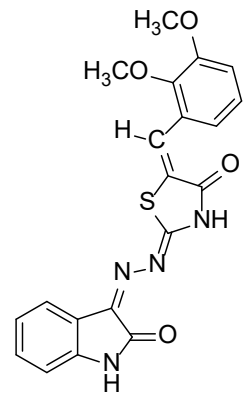


Formula, $\mathrm{C}_{20} \mathrm{H}_{16} \mathrm{~N}_{4} \mathrm{O}_{4} \mathrm{~S}$;

Yield: 71\%; ${ }^{1} \mathrm{H}-\mathrm{NMR}$ : (DMSO- $\left.d_{6}, 300 \mathrm{MHz}\right): \delta 12.1(\mathrm{~s}, 1 \mathrm{H}, \mathrm{NH})$, 10.8(s,1H, NH), 7.9 (s, 1H, CH aldehydic), $7.5\left(\mathrm{~d}, 2 \mathrm{H}, J_{4,5 / 7,6}=7.1 \mathrm{~Hz}\right.$, $\mathrm{H}-4 / 7), 7.3(\mathrm{~m}, 2 \mathrm{H}, \mathrm{H}-5 / 6), 7.1\left(\mathrm{~d}, 1 \mathrm{H}, \mathrm{J}_{2,3^{\prime}}=7.5 \mathrm{~Hz}, \mathrm{H}-2^{\prime}\right), 7.0(\mathrm{~m}, 2 \mathrm{H}$, $\left.\mathrm{H}-3^{\prime} / 4^{\prime}\right)$.

EI-MS: $m / z$ (rel. int. \%): 408(M+1 58), 335(81), 192(100), 172(36).

(2Z,5E)-5-(4-chloro-3-hydroxybenzylidene)-2-((Z)-(2oxoindolin-3 ylidene)hydrazono)thiazolidin-4-one (9)

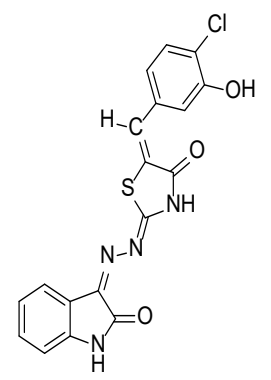

Formula, $\mathrm{C}_{18} \mathrm{H}_{11} \mathrm{ClN}_{4} \mathrm{O}_{3} \mathrm{~S}$;

Yield: 75\%; ${ }^{1} \mathrm{H}-\mathrm{NMR}$ : (DMSO- $\left.d_{6}, 300 \mathrm{MHz}\right): \delta 12.2(\mathrm{~s}, 1 \mathrm{H}, \mathrm{NH})$, 10.6(s,1H, NH), 8.1 (s, $1 \mathrm{H}, \mathrm{CH}$ aldehydic), $7.3\left(\mathrm{~d}, 2 \mathrm{H}, J_{4,5 / 7,6}=7.5 \mathrm{~Hz}\right.$, $\mathrm{H}-4 / 7), 7.2(\mathrm{~m}, 2 \mathrm{H}, \mathrm{H}-5 / 6), 7.1\left(\mathrm{~d}, 1 \mathrm{H}, J_{5^{\prime} 6^{\prime}}=7.5 \mathrm{~Hz}, \mathrm{H}-2^{\prime}\right), 7.0(\mathrm{~m}, 2 \mathrm{H}$, $\left.\mathrm{H}-2^{\prime} / 6^{\prime}\right)$.

EI-MS: $m / z$ (rel. int. \%): 398( $\left.\mathrm{M}^{+}, 88\right), 265(71), 187(100)$, 107(56).

$(2 Z, 5 E)-5-(2,3-d i h y d r o x y b e n z y l i d e n e)-2-((Z)-(2-o x o i n d o l i n-$ 3ylidene)hydrazono)thiazolidin-4-one (10)

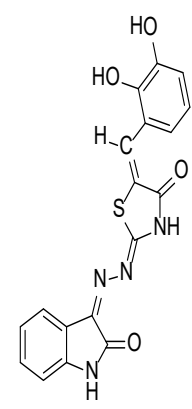

Formula, $\mathrm{C}_{18} \mathrm{H}_{12} \mathrm{~N}_{4} \mathrm{O}_{4} \mathrm{~S}$;

Yield: 67\%; ${ }^{1} \mathrm{H}-\mathrm{NMR}:\left(\mathrm{DMSO}-\mathrm{d}_{6}, 300 \mathrm{MHz}\right): \delta 12.3(\mathrm{~s}, 1 \mathrm{H}, \mathrm{NH})$, 10.3(s,1H, NH), 10.1(s, 2H, OH), 8.1 (s, 1H, CH aldehydic), 7.5 (d, $\left.2 \mathrm{H}, J_{4,5 / 7,6}=7.3 \mathrm{~Hz}, \mathrm{H}-4 / 7\right), 7.3(\mathrm{~m}, 2 \mathrm{H}, \mathrm{H}-5 / 6), 7.2\left(\mathrm{~d}, 1 \mathrm{H}, J_{6,5^{\prime}}=7.1 \mathrm{~Hz}\right.$, H-6'), 7.1(m, 2H, H-4'/5').

EI-MS: $m / z$ (rel. int. \%): 380(M+ $\left.\mathrm{M}^{+}, 88\right), 265(75), 167(100)$, 117(56).

$(2 Z, 5 E)-5-(2-h y d$ roxybenzylidene)-2-((Z)-(2-oxoindolin-3ylidene)hydrazono)thiazolidin-4-one (11)<smiles>O=C1Nc2ccccc2/C1=N/N=C1/NC(=O)/C(=C\c2ccccc2O)S1</smiles>

Formula, $\mathrm{C}_{18} \mathrm{H}_{12} \mathrm{~N}_{4} \mathrm{O}_{3} \mathrm{~S}$;

Yield: 65\%; ${ }^{1} \mathrm{H}-\mathrm{NMR}$ : (DMSO- $\left.d_{6}, 300 \mathrm{MHz}\right): \delta$ 12.1(s, 1H, NH), 10.5(s,1H, NH), 10.1(s, 1H, OH), 8.0 (s, 1H, CH aldehydic), 7.3 (d, $\left.2 \mathrm{H}, J_{4,5 / 7,6}=7.5 \mathrm{~Hz}, \mathrm{H}-4 / 7\right), 7.2\left(\mathrm{~d}, 1 \mathrm{H}, J_{6,5^{\prime}}=7.5 \mathrm{~Hz}, \mathrm{H}-6^{\prime}\right), 7.1(\mathrm{~m}, 3 \mathrm{H}$, $\left.\mathrm{H}-5 / 6 / 3^{\prime}\right), 7.0\left(\mathrm{~m}, 2 \mathrm{H}, \mathrm{H}-4^{\prime} / 5^{\prime}\right)$.

EI-MS: $m / z$ (rel. int. \%): 364(M+1 $\left.\mathrm{M}^{+}, 83\right), 265(85), 162(100)$, $127(56)$

(2Z,5E)-5-(2,4-dichlorobenzylidene)-2-((Z)-(2-oxoindolin-3ylidene)hydrazono)thiazolidin-4-one (12)

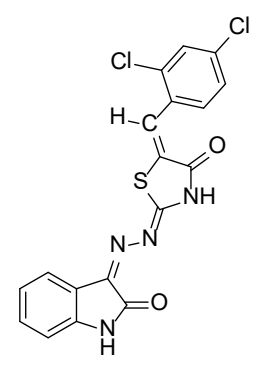

Formula, $\mathrm{C}_{18} \mathrm{H}_{10} \mathrm{Cl}_{2} \mathrm{~N}_{4} \mathrm{O}_{2} \mathrm{~S}$;

Yield: 65\%; ${ }^{1} \mathrm{H}-\mathrm{NMR}$ : (DMSO- $d_{6}, 300 \mathrm{MHz}$ ): $\delta$ 12.2(s, 1H, NH), 10.1(s,1H, NH), 8.2 (s, 1H, H-3'), 8.0 (s, 1H, CH aldehydic), 7.5 (d, $\left.2 \mathrm{H}, J_{4,5 / 7,6}=7.3 \mathrm{~Hz}, \mathrm{H}-4 / 7\right), 7.3\left(\mathrm{~d}, 1 \mathrm{H}, J_{5,6}=7.5 \mathrm{~Hz}, \mathrm{H}-5^{\prime}\right), 7.2(\mathrm{~d}, 1 \mathrm{H}$, $\left.J_{6,5^{\prime}}=7.5 \mathrm{~Hz}, \mathrm{H}-6^{\prime}\right), 7.1(\mathrm{~m}, 2 \mathrm{H}, \mathrm{H}-5 / 6)$;

EI-MS: $m / z$ (rel. int. \%): 415(M+1 $\left.\mathrm{M}^{+}, 83\right), 362(55), 267(100)$, 145(34)

$(2 Z, 5 E)-5$-(4-(dimethylamino)benzylidene)-2-((Z)-(2oxoindolin-3- ylidene)hydrazono)thiazolidin-4-one (13)

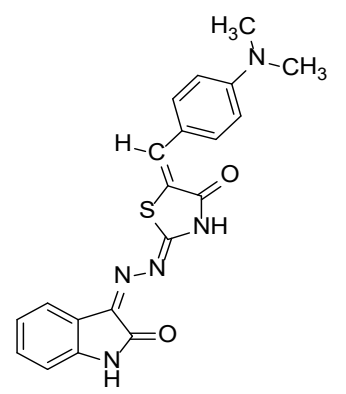

Formula, $\mathrm{C}_{20} \mathrm{H}_{17} \mathrm{~N}_{5} \mathrm{O}_{2} \mathrm{~S}$;

Yield: 82\%; ${ }^{1} \mathrm{H}-\mathrm{NMR}:\left(\mathrm{DMSO}-\mathrm{d}_{6}, 300 \mathrm{MHz}\right.$ ): $\delta$ 12.1(s, 1H, NH), $10.1(\mathrm{~s}, 1 \mathrm{H}, \mathrm{NH}), 8.1$ (s, $1 \mathrm{H}, \mathrm{CH}$ aldehydic), $7.5\left(\mathrm{~d}, 2 \mathrm{H}, J_{4,5 / 7,6}=7.4 \mathrm{~Hz}\right.$, 
$\mathrm{H}-4 / 7), 7.3(\mathrm{~m}, 2 \mathrm{H}, \mathrm{H}-5 / 6), 7.1\left(\mathrm{~d}, 2 \mathrm{H}, \mathrm{H}-J_{2^{\prime} 3^{\prime} / 6^{\prime}, 5^{\prime}}=7.3 \mathrm{~Hz}, \mathrm{H}-2^{\prime} / 6^{\prime}\right)$, $7.0\left(\mathrm{~d}, 2 \mathrm{H}, J_{3^{\prime} 2^{\prime} / 5^{\prime}, 6^{\prime}}=7.5 \mathrm{~Hz}, \mathrm{H}-3^{\prime} / 5^{\prime}\right), 3.2\left(\mathrm{~s}, 6 \mathrm{H}, \mathrm{NCH}_{3}\right)$;

EI-MS: m/z (rel. int. \%): 391(M+1 83), 265(85), 162(100), 127(56).

\section{Lipoxygenase Activity}

Compounds 77-89 were evaluated for Lipoxygenase inhibitory potential. Neither of the compounds showed any variable degree of Lipoxygenase inhibition. The inhibitory potential of the all synthesized compounds is too much less, that is why the IC50 value is not calculated for the compounds [22] (Table 2).

Table 2: Lipoxygenase activity of Isatin derivatives.

\begin{tabular}{|c|c|c|}
\hline S.No. & Inhibition (\%) at $0.25 \mathrm{mM}$ & $\mathrm{IC}_{50}(\mu \mathrm{M})$ \\
\hline 77 & $29.93 \pm 0.26$ & - \\
\hline 78 & $28.64 \pm 0.15$ & - \\
\hline 79 & $13.89 \pm 0.15$ & - \\
\hline 80 & $34.65 \pm 0.18$ & - \\
\hline 81 & $21.42 \pm 0.18$ & - \\
\hline 82 & $35.13 \pm 0.17$ & - \\
\hline 83 & $31.61 \pm 0.27$ & - \\
\hline 84 & $34.56 \pm 0.27$ & - \\
\hline 85 & $11.57 \pm 0.17$ & - \\
\hline 86 & $34.25 \pm 0.19$ & - \\
\hline 87 & $29.69 \pm 0.21$ & - \\
\hline 88 & $9.23 \pm 0.14$ & - \\
\hline 89 & $29.34 \pm 0.15$ & - \\
\hline Baicalein & $93.79 \pm 1.27$ & $22.41 \pm 1.3$ \\
\hline
\end{tabular}

\section{Conclusion}

Here, we have explored heterocyclic compounds having one or more different atoms like sulphur, nitrogen and oxygen in their ring skeleton instead of carbon ranging in size from three members ring to higher members ring. Synthesis of small, simple heterocyclic molecules in search of lead molecules is the basic aim of our research study. Thiazole and Isatin have great biological importance. Due to the great biological importance here we have design a project on synthesis of a hybrid molecule of this two important molecules i.e isatin \& thiazole and their biological evaluation with the assumption that this molecule might have more potential.

\section{Compliance with Ethical Standards}

Conflict of interest: The authors declare that they have no conflict of interest.

\section{Ethical Approval}

This article does not contain any studies with human participants or animals performed by any of the authors.

\section{Acknowledgements}

This research was funded by Department of Chemistry, Hazara University, Mansehra 21300, Pakistan.

\section{References}

1. A Medvedev, O Buneeva, O Gnedenko, P Ershov, A Ivanov (2018) Isatin an endogenous nonpeptide biofactor: A review of its molecular targets, mechanisms of actions, and their biomedical implications. Bio Factors 44(2): 95-108.

2. R Mishra, A Jana, AK Panday, LH Choudhury (2019) Synthesis of spirooxindoles fused with pyrazolo-tetrahydropyridinone and coumarin-dihydropyridine-pyrazole tetracycles by reaction medium dependent isatin-based multicomponent reactions. New J Chem 43(7): 2920-2932.

3. R Sabet, M Mohammadpour, A Sadeghi, A Fassihi (2010) QSAR study of isatin analogues as in vitro anti-cancer agents. Eur J Med Chem 45: 1113 1118.

4. L Zhang, W Sun, Y Cao, L Hou, C Ju, et al. (2019) Isatin inhibits the invasion of human neuroblastoma SH-SY5Y cells, based on microarray analysis. Mol Med Rep 20(2): 1700-1706.

5. Varun, Sonam, R Kakkar (2019) Isatin and its derivatives: A survey of recent syntheses, reactions, and applications. Medchemcomm 10(3): 351-368.

6. V Fernandez Moreira, C Val Campillo, I Ospino, RP Herrera, I Marzo, et al. (2019) Bioactive and luminescent indole and isatin based gold(i) derivatives. Dalton T 48(9): 3098-3108.

7. H Hou, H Li, Y Han, C Yan (2018) Synthesis of visible-light mediated tryptanthrin derivatives from isatin and isatoic anhydride under transition metal-free conditions. Org Chem Front 5(1): 51-54.

8. S Khabnadideh, R Sabet, HP Naghz, M Divar (2019) Quantitative Structure Activity Relationship of New Isatin Analogues for Design New Compounds as Anti-breast Cancer. J Pharm Res Int 28(5): 1-16.

9. M Franc, M Urban, I Císařová, J Veselý (2019) Highly enantioselective addition of sulfur-containing heterocycles to isatin-derived ketimines. Org Biomol Chem 17(31): 7309-7314.

10. H Pellissier (2018) Synthesis of chiral 3-substituted 3-amino-2oxindoles through enantioselective catalytic nucleophilic additions to isatin imines. Beilstein J Org Chem 14: 1349-1369.

11. B Sharma, A Singh, L Gu, ST Saha, A Singh Pillay, et al. (2019) Diastereoselective approach to rationally design tetrahydro- $\beta$-carbolineisatin conjugates as potential SERMs against breast cancer. Rsc Adv 9(17): 9809-9819.

12. A0 Abdelhamid, SM Gomha (2017) Synthesis and characterization of new pyrazole-based thiazoles. Synthetic Communications 47(15): 14091414

13. AA Fadda, ESM Afsah, RS Awad (2013) Synthesis and antimicrobial activity of some new benzo and naphthonitrile derivatives. Eur J Med Chem 60: 421-430.

14. N Zelisko, D Atamanyuk, O Vasylenko, P Grellier, R Lesyk (2012) Synthesis and antitrypanosomal activity of new 6,6,7-trisubstituted thiopyrano 2,3-d 1,3 thiazoles. Bioorg Med Chem Lett 22(23): 7071-7074.

15. L Yang, J Zhao, X Yang, M Chen, Y Xue (2019) Effects of solvents on the DACBO-catalyzed vinylogous Henry reaction of isatin with 3,5-dimethyl4-nitroisoxazole "on-water" and in solution from QM/MM MC simulations. Rsc Adv 9(9): 4932-4941.

16. C Yin, C Xu, W Yu, Y Jia, W Sun, et al. (2019) Synthesis of a novel isatin and ethylenediamine modified resin and effective adsorption behavior towards Orange G. Rsc Advances 9(2): 801-809. 
17. J Kaur, SS Chimni (2018) Catalytic synthesis of 3-aminooxindoles via addition to isatin imine: An update. Org Biomol Chem 16(18): 33283347.

18. M Ahmed, M Arif, F Jabeen, H Khan, K Ansar, et al. (2019) On the importance of antiparallel $\pi-\pi$ interactions in the solid state of isatinbased hydrazides. New J Chem.

19. Z Sun, K Xiang, H Tao, L Guo, Y Li (2018) Synthesis of 2-substituted 3-chlorobenzofurans via TMSCl-mediated nucleophilic annulation of isatin-derived propargylic alcohols. Org Biomol Chem 16(33): 61336139.

ISSN: 2574-1241

DOI: $10.26717 / B J S T R .2020 .28 .004704$

Tariq Aziz. Biomed J Sci \& Tech Res

This work is licensed under Creative Commons Attribution 4.0 License

Submission Link: https://biomedres.us/submit-manuscript.php
20.JY Zhu, WL Yang, YZ Liu, SJ Shang, WP Deng (2018) A copper(I)catalyzed asymmetric Mannich reaction of glycine Schiff bases with isatin-derived ketimines: Enantioselective synthesis of 3-substituted 3-aminooxindoles. Org Chem Front 5(1): 70-74.

21. S Hu, J Zhang, Q Jin (2018) DMAP-catalyzed alkylation of isatin N,N' cyclic azomethine imine 1,3-dipoles with Morita-Baylis-Hillman carbonates. New J Chem 42(9): 7025-7029.

22. JJ Ji, H Yan, P Xiang, BH Shen, M Shen (2019) An LC-MS/MS method for the simultaneous determination of 12 psychotropic drugs and metabolites in hair: Identification of acute quetiapine poisoning using hair root. Forensic Sci Int 301: 341-349.

$\begin{array}{ll}\text { BIOMEDICAL } & \text { Assets of Publishing with us } \\ \text { RESEARCHES } & \text { - Global archiving of articles } \\ & \text { - Immediate, unrestricted online access } \\ \end{array}$

